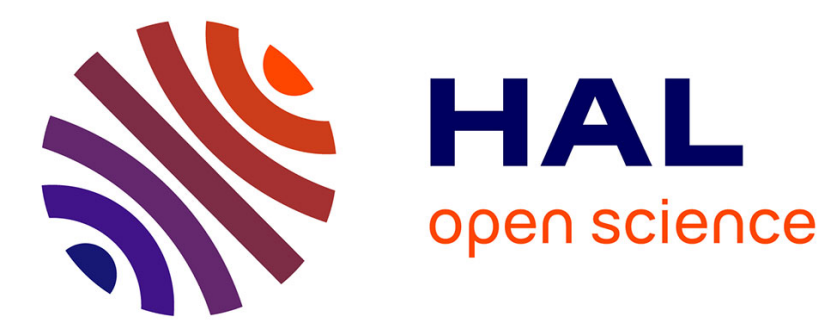

\title{
Coupling into slow-mode photonic crystal waveguides
}

Jean-Paul Hugonin, Philippe Lalanne, Thomas P. White, Thomas F. Krauss

\section{To cite this version:}

Jean-Paul Hugonin, Philippe Lalanne, Thomas P. White, Thomas F. Krauss. Coupling into slow-mode photonic crystal waveguides. Optics Letters, 2007, 32 (18), pp.2638-2640. 10.1364/OL.32.002638 . hal-00880160

\section{HAL Id: hal-00880160 \\ https://hal-iogs.archives-ouvertes.fr/hal-00880160}

Submitted on 5 Nov 2013

HAL is a multi-disciplinary open access archive for the deposit and dissemination of scientific research documents, whether they are published or not. The documents may come from teaching and research institutions in France or abroad, or from public or private research centers.
L'archive ouverte pluridisciplinaire HAL, est destinée au dépôt et à la diffusion de documents scientifiques de niveau recherche, publiés ou non, émanant des établissements d'enseignement et de recherche français ou étrangers, des laboratoires publics ou privés. 


\title{
Coupling into slow-mode photonic crystal waveguides
}

\author{
J. P. Hugonin, ${ }^{1}$ P. Lalanne, ${ }^{1, *}$ T. P. White, ${ }^{2}$ and T. F. Krauss ${ }^{2}$ \\ ${ }^{1}$ Laboratoire Charles Fabry de l'Institut d'Optique, CNRS, Univ Paris-Sud, Campus Polytechnique, \\ RD 128, 91127 Palaiseau Cedex, France \\ ${ }^{2}$ School of Physics and Astronomy, University of St Andrews, St Andrews, Fife, KY169SS, UK \\ *Corresponding author: philippe.lalanne@institutoptique.fr
}

Received May 14, 2007; revised July 17, 2007; accepted July 24, 2007;

posted July 30, 2007 (Doc. ID 82885); published September 4, 2007

\begin{abstract}
We study efficient injectors for coupling light from $z$-invariant ridge waveguides into slow Bloch modes of single-row defect photonic crystal waveguides. Two-dimensional vectorial computations performed with a Bloch mode theory approach predict that very high efficiencies $(>90 \%)$ can be achieved for injector lengths of only a few wavelengths in length, even for small group velocities in the range of $c / 100-c / 400$. This result suggests that photonic crystal devices operating with slow waves can be interfaced with classical waveguides without sacrificing compactness. (C) 2007 Optical Society of America OCIS codes: $130.2790,350.7420$.
\end{abstract}

Planar two-dimensional (2D) slab-type photonic crystals have attracted much attention recently as a possible platform for densely integrated photonic circuits and novel photonic functionality. Engineering the photonic dispersion relationship might provide unique opportunities for integrated photonics, especially in the slow-light regime where new compact devices, such as delay lines, optical switches, or Raman lasers, are currently the subject of intense research [1-7]. However, an increase of the group index $n_{\mathrm{g}}$ in the slow-light regime prevents efficient coupling because of increasingly large mode mismatch. Thus the realization of efficient and compact injectors is a crucial component for further studies in this field [6,8-10].

In this Letter we study efficient and compact injection into slow modes supported by single-row-defect photonic crystal waveguides (PhCWs). In contrast with previous studies related with injectors operating at moderate slowdown factors $[9,10]$, we target much stronger slowdown factors with $n_{\mathrm{g}}$ in the range of one to several hundreds. For such factors, which are important for reaching strong nonlinear interaction regimes, one generally anticipates weak injection efficiencies. We show here that $\mathrm{PhCWs}$ naturally offer efficient injection and that coupling efficiencies of $95 \%$ might be achieved for $n_{\mathrm{g}} \approx 200-400$ with couplers that are only a few wavelengths long. For this, we first explore the possibility of improving mode matching and obtaining efficient coupling between fast and slow PhCW modes. Further, we design compact couplers between classical $z$-invariant ridge waveguides and slow PhCWs by first coupling the light from the ridge into the fast mode of a PhCW and then into the slow PhCW mode.

We start by considering the coupling between slowand fast-light $\mathrm{PhCWs}$. In photonic crystal circuit platforms, such couplers can be used for locally slowing down the light, or by virtue of Bloch mode reciprocity [11], to accelerate it. Figure 1(a) shows an abrupt interface between such waveguides. The top waveguide is a single-row-defect PhCW (W1) with a lattice constant of $a=420 \mathrm{~nm}$ and a hole radius of $r$ $=0.3 a$. It supports a slow Bloch mode for frequencies $a / \lambda$ just above the cutoff frequency $a / \lambda=0.260(\lambda$ $=1.616 \mu \mathrm{m})$. The bottom $\mathrm{PhCW}\left(\mathrm{W}^{\prime}\right)$ is obtained from the W1 waveguide by increasing the period along the $z$ direction to $a^{\prime}=450 \mathrm{~nm}$. As shown by the dispersion diagrams in Fig. 1(b), the $\mathrm{W}^{\prime}$ waveguide supports a fast Bloch mode $\left(n_{\mathrm{g}}^{\prime} \approx 5\right)$ in the slow-mode regime of the $\mathrm{W} 1$ waveguide.

We have studied the design of efficient injectors from $\mathrm{W}^{\prime}$ to $\mathrm{W} 1$. The calculation is performed with a Bloch mode Fourier modal method. The modal reflection and transmission coefficients at the interface are obtained analytically by using a quasi-normal Bloch basis [11] and by matching the tangential electromagnetic field components at the interface. To ensure accuracy, especially for large $n_{\mathrm{g}}$, the calculation is performed by assuming that the geometry is invariant in the $y$ direction (2D calculation), using an effective index of 2.83 for the refractive index value of the semiconductor and a magnetic polarization parallel to the $\mathrm{PhC}$ cylinder $y$ axis. The effective index value was chosen so that the cutoff frequency of the W1 waveguide at the edge of the first Brillouin zone matches that of a real 3D structure etched in a silicon
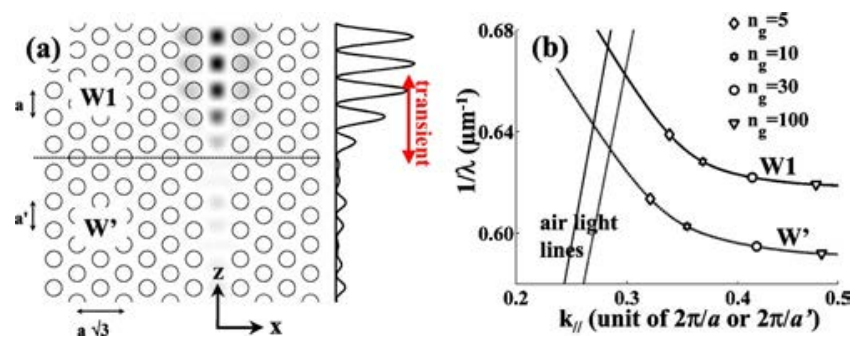

Fig. 1. (Color online) Interface between two PhCWs supporting slow (W1) and fast ( $\left.\mathrm{W}^{\prime}\right)$ Bloch modes at the same frequencies. (a) $\left|H_{\mathrm{y}}\right|^{2}$ distribution for $\lambda=1.615 \mu \mathrm{m} \quad\left(n_{\mathrm{g}}\right.$ $=100$ ). The right inset is a cut of $\left|H_{y}\right|^{2}$ on the waveguide $z$ axis. (b) Dispersion diagram of the fundamental guided Bloch modes of the waveguides. The lines represents the air light lines of the two waveguides. 
membrane with a $240 \mathrm{~nm}$ thickness. To avoid any interference induced by the coupling of adjacent line defects through the surface states possibly excited along the interface, we use perfectly matched layers implemented as complex nonlinear transforms for terminating the computational window in the transverse $x$ direction.

In the absence of any taper between the two waveguides of Fig. 1, the coupling efficiency $T$ from $\mathrm{W}^{\prime}$ to $\mathrm{W} 1$ decreases monotonically as the 12 th power of $\left(n_{\mathrm{g}}-n_{\mathrm{g}}^{\prime}\right) /\left(n_{\mathrm{g}}+n_{\mathrm{g}}^{\prime}\right)$ [dashed curve in Fig. 2(c)]. In comparison with previous theoretical work predicting $\approx 3-5 \%$ injection efficiencies at $n_{\mathrm{g}}=100$ for $1 \mathrm{D}$ periodic thin film stacks or related systems relying on patterned waveguides $[5,8]$, the coupling is rather efficient in the present situation, reaching a value as high as $80 \%$ for $n_{\mathrm{g}}=100$. To explain this surprisingly large efficiency, we have calculated the field pattern for a wavelength of $3.845 a\left(n_{g}=100\right)$. The squared modulus of the magnetic field $H_{\mathrm{y}}$ is superimposed in Fig. 1(a). The remarkable observation apparent from Fig. 1(a) is that, although it is facing an abrupt interface between two waveguides (without any taper), the field experiences a transient zone, where it smoothly varies as it penetrates into the $\mathrm{W} 1$ waveguide. As shown on the right side of the figure, the intensity pattern increases progressively in the first two periods of the $\mathrm{W} 1$ waveguide and then reaches a
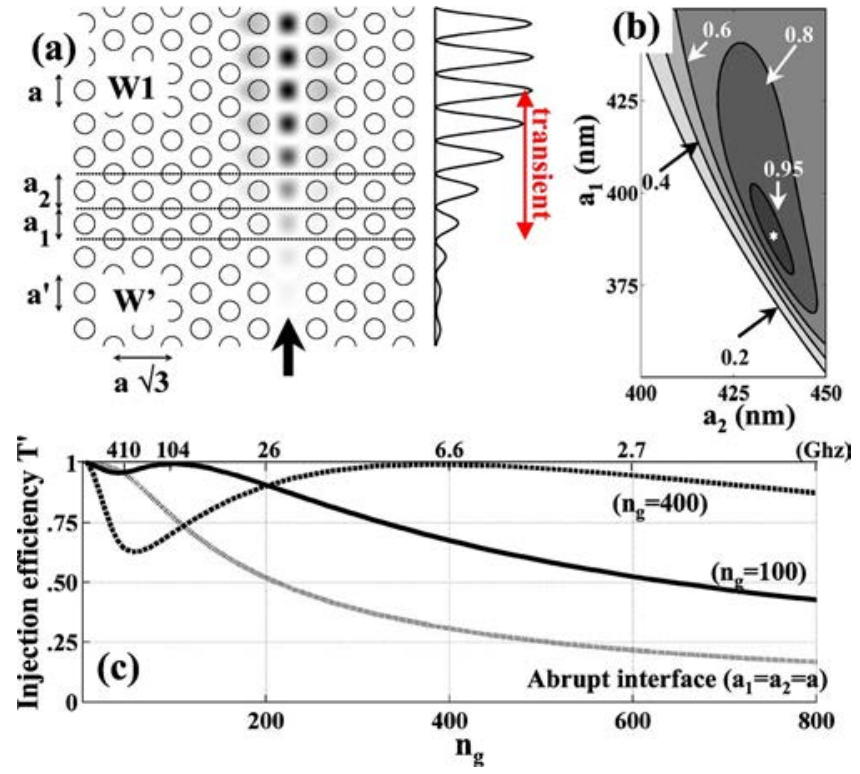

Fig. 2. (Color online) Performance of a two-cell injector heterostructure for coupling between fast and slow PhCW Bloch modes. (a) Sketch of the two-cell geometry. The background represents the $\left|H_{\mathrm{y}}\right|^{2}$ distribution calculated for an incident illumination from the fundamental $\mathrm{W}^{\prime}$ Bloch mode at $\lambda=1.615 \mu \mathrm{m}\left(n_{\mathrm{g}}=100\right)$. (b) Tolerance of the coupling efficiency $T^{\prime}$ as a function of the periods, $a_{1}$ and $a_{2}$, of the injector for $\lambda=1.615 \mu \mathrm{m}\left(n_{\mathrm{g}}=100\right)$. (c) Coupling efficiency as a function of the group index of the slow W1 PhC-Bloch mode. Solid curve, $a_{1}=0.925 a$ and $a_{2}=1.038 a\left(n_{\mathrm{g}}=100\right)$, as marked with a star in (b); dotted curve, $a_{1}=0.867 a$ and $a_{2}$ $=1.064 a\left(n_{\mathrm{g}}=100\right)$; dashed curve, abrupt interface $\left(a_{1}=a_{2}\right.$ $=a)$. The top horizontal scale represents the distance in $\mathrm{GHz}$ from the cutoff frequency, $\lambda=1.616 \mu \mathrm{m}$. stationary value that is approximately $n_{\mathrm{g}} / n_{\mathrm{g}}^{\prime}$ times larger than that of the $\mathrm{W}^{\prime}$ waveguide. We emphasize that the existence of a transient zone is absolutely required for efficient coupling [8] and that this zone is obtained for an abrupt interface without any adiabatic transition. In our Bloch mode Fourier modal computations, the transient zone results from an interference pattern that combines several W1 Bloch modes, the fundamental propagating Bloch mode of Fig. 1(b), and three other evanescent (nonpropagating) Bloch modes with complex propagation constants. Note that the coexistence of several evanescent and propagative Bloch modes in periodic Bragg waveguides has been recently discussed, and this has been shown to strongly affect the $Q$ factor of micropillars [12].

Encouraged by this result, we have engineered the interface between the $\mathrm{W}^{\prime}$ and $\mathrm{W} 1$ waveguides to optimize the injection efficiency. Figure 2 shows the improvement available by adding suitable interface layers. By optimizing the added degrees of freedom, namely the two layer periodicities $a_{1}$ and $a_{2}$ [Fig. 2(a)], a very good coupling efficiency over a broad range of group velocities can be achieved for almost any desired value of the group indices; we discuss here the cases of $n_{\mathrm{g}}=100$ and $n_{\mathrm{g}}=400$ as two examples. For the design, we have explored the parameter space spanned by $350 \mathrm{~nm}<a_{1}<450 \mathrm{~nm}$ and $400 \mathrm{~nm}<a_{2}<450 \mathrm{~nm}$. Figure 2(b) shows the injection efficiency $T^{\prime}$ of the engineered interface as a function of $a_{1}$ and $a_{2}$ for $n_{\mathrm{g}}=100$. In comparison with the abrupt interface case $(T=78 \%), T^{\prime}$ peaks at a much larger value of $99.7 \%$ obtained for the star mark $\left(a_{1}=0.925 a\right.$ and $\left.a_{2}=1.038 a\right)$. Note that since $a_{1}<a$, the optimum $a_{1}$ layer does not support any propagating Bloch mode and basically acts as a mirror. This result is consistent with previous injector designs that promote the use of mirror-type injectors [8]. In addition, we note that the design tolerance is compatible with existing fabrication techniques. In Fig. 2(a), we have superimposed the squared modulus of the magnetic field distribution calculated for the optimal values of the periodicities and for $n_{\mathrm{g}}$ $=100$. The field intensity gradually increases, and in comparison with Fig. 1(a), it is found that the transient zone is larger $(\approx 5$ periods $)$. The injector clearly helps to smooth out the field transition. Figure 2(c) shows the coupling efficiency $T^{\prime}$ (solid curve) as a function of the group index of the W1 waveguide Bloch mode. A marked improvement is achieved in comparison with the abrupt interface case (dashed curve). Similar optimizations have been performed at $n_{\mathrm{g}}=400$. Again, the calculation has shown that almost perfect injection $\left(T^{\prime}=99.5 \%\right)$ can be achieved for an injector with $a_{1}=0.867 a$ and $a_{2}=1.064 a$. The performance of this injector is shown by the dotted curve in Fig. 2(c).

The $-1 \mathrm{~dB}\left(T^{\prime}>0.8\right)$ bandwidth of the injector can be estimated from Fig. 2(c) by using the top horizontal scale shown in GHz. Actually, the injector designed for optimal operation at $n_{\mathrm{g}}=100$ offers bandwidths of 600 and $220 \mathrm{GHz}$ when operated at 
wavelengths corresponding to $n_{\mathrm{g}}=50$ and 100 , respectively. The bandwidth is broad enough to support high-data-rate telecommunication signals. Indeed, as the operating wavelength is further reduced, the bandwidth that vanishes as $\left(n_{\mathrm{g}}\right)^{-2}$ is ultimately limited by the mode cutoff at the boundary of the Brillouin zone. We believe that $n_{\mathrm{g}}$ values in the range of 50-100 may represent an interesting regime for onchip optical processing, while larger $n_{\mathrm{g}}$ may find applications for single-frequency applications in nonlinear optics [2] or in new architectures for lasing applications [13].

For practical applications, coupling from a ridge waveguide that only supports a truly guided fundamental mode with a large group velocity into a slow Bloch mode is essential. Figure 3 shows such a situation, where the light impinging from the ridge (bottom) is first coupled into the fast $\mathrm{W}^{\prime}$ waveguide and then into the slow W1 Bloch mode through the injector studied in Fig. 2. Two transmission coefficients are mainly involved in the coupling process, $\mathrm{T}^{\prime}$ as defined previously, and $\mathrm{T}^{\prime \prime}$ the coupling between the ridge and $\mathrm{W}^{\prime}$. We have first optimized the ridge- $\mathrm{W}^{\prime}$ interface by changing the termination truncation at the interface. Large coupling efficiencies are obtained for $\lambda=1.615 \mu \mathrm{m}$ with the termination shown in Fig. 3 (a). This termination is refered to as $\tau=-0.35$ in the terminology in [6]. As shown by the solid curve in Fig. $3(\mathrm{~b}), T^{\prime \prime} \approx 96 \%$ in the regime of slow-light W1 operation. Figure 3(b) shows the total injection efficiency $T_{\mathrm{t}}$ for different values of the length $\mathrm{Na}^{\prime}$ of the $\mathrm{W}^{\prime}$ section. All calculations have been performed for the injector optimized for $n_{\mathrm{g}}=100$. For small values of $N, T_{\mathrm{t}}$ is rather weak. It is limited not by Fabry-Perot effects involving multiple reflections of the fundamental $\mathrm{W}^{\prime}$ Bloch mode between the two interfaces as one may first expect but by $\mathrm{W}^{\prime}$ evanescent Bloch modes that are similar to those previously discussed and that are excited at the ridge- $\mathrm{W}^{\prime}$ interface. These modes funnel through the $\mathrm{W}^{\prime}$ section and, for small $N$, destructively interfere in the W1 waveguide to lower the total coupling efficiency. This is evidenced
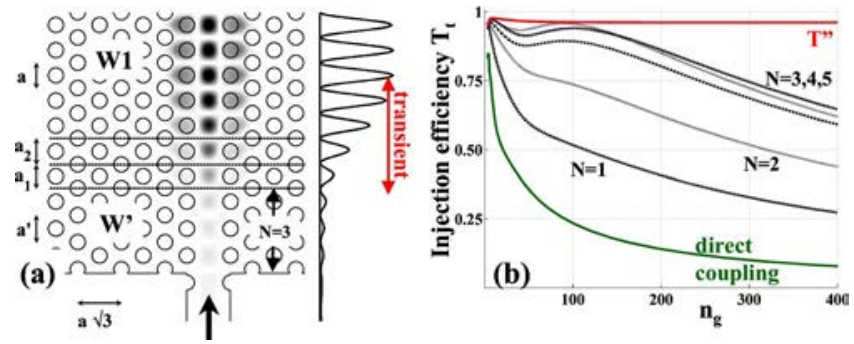

Fig. 3. (Color online) Light injection from a $z$-invariant waveguide into a W1 slow Bloch mode. (a) Two-step injection geometry. $N$ represents the number of periods of the fast $\mathrm{PhCW} \mathrm{W}^{\prime}$ section. The superimposed background is defined as before. (b) Total coupling efficiency $T_{\mathrm{t}}$ as a function of the group index of the slow PhC Bloch mode for different values of $N$, and for $a_{1}=0.925 a$ and $a_{2}=1.038 a$ [star in Fig. 2(b)]. Curve $\mathrm{T}^{\prime \prime}$ represents the coupling efficiency between the ridge and the $\mathrm{W}^{\prime}$ waveguide, and the curve labeled direct coupling represents the coupling efficiency from the ridge waveguide to $\mathrm{W} 1, a^{\prime}=a_{1}=a_{2}=a$ in (a). by considering the values of $T_{\mathrm{t}}$ for $n_{\mathrm{g}}=100$. In this case, $T^{\prime \prime} \approx 96 \%$ and $\mathrm{T}^{\prime}$ is nearly equal to $1\left(T^{\prime}\right.$ $=99.7 \%$ ). The modal reflectivities of the fundamental $\mathrm{W}^{\prime}$ Bloch mode are therefore very small. The FabryPerot effect vanishes and cannot be invoked to explain the variations of $T_{\mathrm{t}}$ as a function of $N$. As $N$ increases, the total coupling efficiency $T_{\mathrm{t}}$ rapidly reaches a stationary regime with $T_{\mathrm{t}} \approx T^{\prime} T^{\prime \prime}$, as shown by the curves obtained for $N=4$ and 5 . Note that for $N=5, T_{\mathrm{t}}=0.96$ is almost equal to $T^{\prime \prime}$ and is indeed limited by the ridge- $\mathrm{W}^{\prime}$ coupling rather than the $\mathrm{W}^{\prime}-\mathrm{W} 1$ coupling. Very similar results not reported here for the benefit of compactness have been obtained for the injector designed for $n_{\mathrm{g}}=400$. To access the out-of-plane scattering losses that are not taken into account into the $2 \mathrm{D}$ simulations, we have additionally performed 3D calculations for the geometry shown in Fig. 3(a) and for a $240 \mathrm{~nm}$ silicon membrane. It turns out that the losses are $\approx 0.06$ and are merely independent of the group velocity for $30<n_{\mathrm{g}}$ $<400$.

In conclusion, we have provided a theoretical study of the coupling efficiency between a ridge and a single-row-defect $\mathrm{PhCW}$ in the slow-light regime. Injection efficiencies close to $96 \%$ are predicted with $2 \mathrm{D}$ computational results for injector lengths of only a few wavelengths and group indices as large as 100400 . We believe that the feasibility of compact and efficient light injectors into slow W1 Bloch modes is due to the existence of evanescent Bloch modes other than the fundamental one to be excited. This property is rather unusual and is not encountered in more conventional periodic systems [5,7].

This research is partly supported under the European contract SPLASH of the 6th EU Framework programme and by the Agence Nationale de la Recherche under contract MIRAMAN of the French ANR Nano2006. T. P. White is supported by an 1851 Royal Commission Research Fellowship.

\section{References}

1. M. F. Yanik and S. Fan, Phys. Rev. Lett. 92, 083901 (2004)

2. M. Soljacic and J. D. Joannopoulos, Nat. Mater. 3, 211 (2004).

3. T. F. Krauss, Phys. World 19(2), 32 (2006).

4. J. Khurgin, Opt. Lett. 30, 2778 (2005).

5. M. L. Povinelli, S. Johnson, and J. Joannopoulos, Opt. Express 13, 7145 (2005).

6. Y. A. Vlasov and S. J. McNab, Opt. Lett. 31, 50 (2006).

7. H. Rong, R. Jones, A. Liu, O. Cohen, D. Hak, A. Fang, and M. Paniccia, Nature 433, 725 (2005).

8. P. Velha, J. P. Hugonin, and P. Lalanne, Opt. Express 15, 6102 (2007).

9. N. Ozaki, Y. Kitagawa, Y. Takata, N. Ikeda, Y. Watanabe, A. Mizutani, Y. Sugimoto, and K. Asakawa, Opt. Express 15, 6974 (2007).

10. P. Pottier, M. Gnan, and R. M. De La Rue, Opt. Express 15, 6569 (2007).

11. G. Lecamp, J. P. Hugonin, and P. Lalanne, Opt. Express 15, 11042 (2007).

12. G. Lecamp, P. Lalanne, J. P. Hugonin, and J. M. Gérard, IEEE J. Quant. Electron. 41, 1323 (2005).

13. X. Yang and C. W. Wong, Opt. Express 13, 4723 (2005). 